\title{
Effect of Metallic Objects and Liquid Supplies on RFID Links
}

\author{
Yimin Zhang, Konstantin Yemelyanov, Xin Li, Moeness G. Amin \\ Center for Advanced Communications \\ Villanova University, Villanova, PA 19085 \\ E-mail:\{yimin.zhang, konstantin.yemelyanov, xin.l, moeness.amin\}@villanova.edu
}

\section{Introduction}

This paper studies the effect of nearby metallic objects and liquid supplies, which may be commonly encountered in warehouses, on RFID link characteristics and read range. Such studies are performed in two different fronts. Simulation results using a commercial finite-difference time-domain software package provide detailed views of the effect of metallic objects and liquid supplies on the signal strength. The read range is further examined by experiments.

\section{Background}

An RFID system involves wireless communications between the reader and tags. To maintain the quality of an RFID link, both the forward-link and backward-link should have sufficiently high power level at the tag and reader, respectively. It is known that, for current IC-based passive tags, the read range is forward-linklimited [1]. In the free space, the read range is primarily determined by the path loss, which is a function of the transmit power and the distance between the reader and tag. In practice, however, RFID read range may be affected by the actual propagation channel which is often complicated. In particular, local reflectors and scatterers introduce a multipath problem and yield channel impairments due to fading. In such an environment, signals propagated through different paths may add up either constructively or destructively due to randomly time-varying delays, phases, and attenuations in the various paths.

We have examined the effect of several metallic objects and liquid supplies, commonly found in warehouses, to a nearby RFID system. The studies include both numerical computations and experiments. The results obtained for a metallic cabinet and a stack of water bottles are presented in this paper.

\section{Numerical Simulations}

Scattered electromagnetic filed was calculated using REMCOM XFDTD commercial software package. The dimension of the space considered in the simulations was $5 \times 1 \times 1 \mathrm{~m}$ (range $\mathrm{x}$ width $\mathrm{x}$ height). The incident field was produced by a half-wavelength dipole with the center frequency of $915 \mathrm{MHz}$. The dipole was parallel to the $z$-axis and its center is at located at (range) $x=0$ $\mathrm{m}$, (width) $y=0.5 \mathrm{~m}$, and (height) $z=0.5 \mathrm{~m}$. The amplitude of the received signal was converted to the receive signal power $(\mathrm{dBm})$ assuming the tag as an 
isotropic antenna. The transmitted power was $1 \mathrm{~W}$ in all the cases presented here. It should be noted that FCC regulations allow a maximum transmit power of $1 \mathrm{~W}$, provided that the effective isotropic radiated power (EIRP) does not exceed $6 \mathrm{dBi}$. Typical IC-based UHF passive RFID tags require a receive power level of -15 to $-10 \mathrm{dBm}$ to maintain a reliable downlink [1].

\section{A. Empty Scene}

The received signal power in the $x-y$ plane for the empty scene scenario is given Figure 1. This serves as a baseline for the other scenarios considered in the paper.

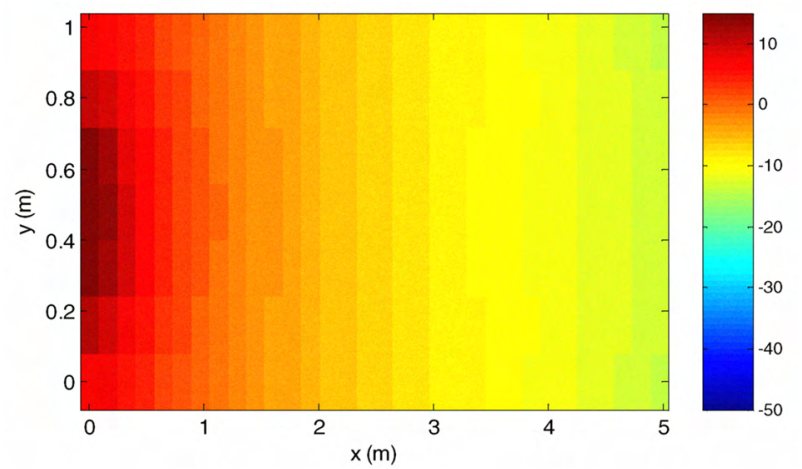

Figure 1 Received signal power for the free space scenario

\section{B. Effect of Metallic Cabinet}

We considered the effect of a metallic cabinet placed at two different positions around the RFID system, namely, at the side of the link path and in front of the reader (emulating the scenario when the cabinet is located behind the tag). The dimensions of the cabinet were approximately $0.67 \times 0.38 \times 0.89 \mathrm{~m}$ (width $\mathrm{x}$ depth $\mathrm{x}$ height).

It is observed (see the left plot of Figure 2) that the received signal power level behind the cabinet is significantly reduced due to obstruction. The level of the received signal power around the cabinet is also affected, which yields a nonmonotonic variation in the power level in the range direction. The oscillating power level for the case when the distance between the cabinet and the reader along the $x$-axis is $5 \mathrm{~m}$ is presented in the right plot of Figure 2. One may notice that the power of the received signal in the area close to the cabinet changes rapidly. Such variations, caused by reflections from the cabinet surface, range 20 $\mathrm{dB}$. Similar phenomena were observed when a reader is close to the cabinet.

\section{Effect of Liquid Bottles}

The second object of interest was a stack of bottled water cases placed at two different locations around the RFID system in a way similar to those for the metallic cabinet. Five cases, each containing twenty-four $500 \mathrm{ml}$ bottles of water, stacked on top of each other. The power levels of the received signal for the two scenarios are presented in Figure 3. The results are, in general, similar to those for the metallic cabinet, but their fading patterns slightly differ. 


\section{Experimental Results}

Experiments were performed in a classroom of approximately $9.5 \times 8 \mathrm{~m}$ using the Psion Teklogic Workabout Pro Handheld Reader with RFID module option [2]. Three types of RFID tags were used in the tests, namely, Avery AD-222 (type 1), Avery AD-820 (type 2), and Omron V750 Wave (type 3). In the tests, the tags were attached to a carton box. The reader and the carton box were located on the top of separate plastic containers of approximately the same height.

In order to examine the effect of metallic cabinet and liquid bottles, five different scenarios were considered:

- "At-a-side": the closest corner of the object was shifted from the center of the RFID reader antenna by $1 \mathrm{~m}$ in range direction and by $0.3 \mathrm{~m}$ in cross-range direction (one scene).

- "Behind-the-reader": the object was located behind the reader at the two different distances $d$ (two scenes).

- "Behind-the-tag": the object was located behind the tag, at the two different distances $d$ (two scenes).

The read range in the presence the metallic cabinet and water bottles is shown in Figure 4. The "free space" here is the scene without either the metallic cabinet or liquid bottles. It is observed that the effect of the cabinet in the "at-a-side" case is not significant. "Behind-the-reader" cases were measured for the two different values of the distance, namely, $d=50 \mathrm{~cm}$ and $d=39 \mathrm{~cm}$, respectively. The read range in the two cases differs significantly, showing its sensitivity to the reader's position. This effect, previously observed in the numerical simulations, confirms the oscillating nature of the signal level phenomenon. Similar results were obtained for the two different "behind-the-tag" scenarios. The respective distances were the same as those for the "behind-the-reader" cases. The effect of water bottles is similar but less significant, probably because the bottles were not aligned perfectly.

\section{Conclusion}

Metallic objects and liquid supplies around a reader and/or tags may significantly affect the read range of RFID systems. Strong reflecting signals may be generated by the metallic and liquid surface. Depending on the phase of the reflected signal relative to that of the signal transmitted by the reader or reflected by the tag, the reflected signal may affect the signal strength constructively or destructively. Thus, in some situations the read range may be extended due to the reflection, and in other situations the read range may be significantly reduced. The most significant impact is achieved when the metallic objects and liquid supplies are relatively large and close to the reader and/or tag. It is advised that the RFID system is operated away from metallic material and liquid supplies. Multiple RFID antennas may achieve spatial diversity and improve the link reliability over an extended read range. 


\section{Acknowledgments}

Funding for the research was provided in part by Verdasee Solutions (www.verdasee.com), and Telemedicine and Advanced Technology Research Center (TATRC), an agency reporting to the U.S. Army Medical Research and Materiel Command, Ft. Detrick, Maryland. The content of the paper does not necessarily reflect the position or the policy of Verdasee Solutions or the Government, and no official endorsement should be inferred.

\section{References}

[1] D. M. Dobkin, The RF in RFID: Passive UHF RFID in Practice. Boston, MA: Newnes, 2008.

[2] Psion Teklogix, 2nd Generation Workabout Pro Hand-Held Computer, $\mathrm{http}: / / \mathrm{www}$. psionteklogix.com/public.aspx?s=us\&p=Products\&pID=5194.
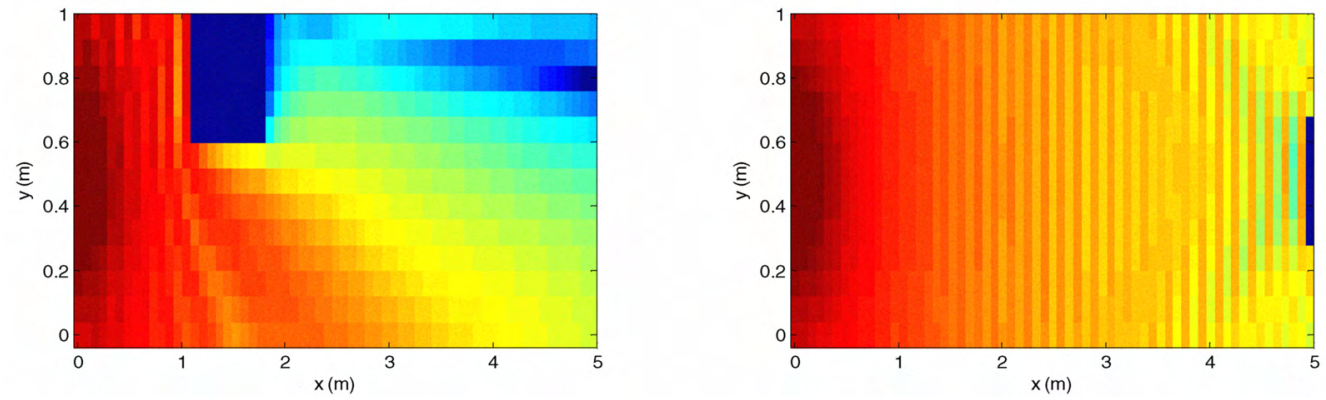

Figure 2 With metallic cabinet (left: at-a-side; right: in front of reader)
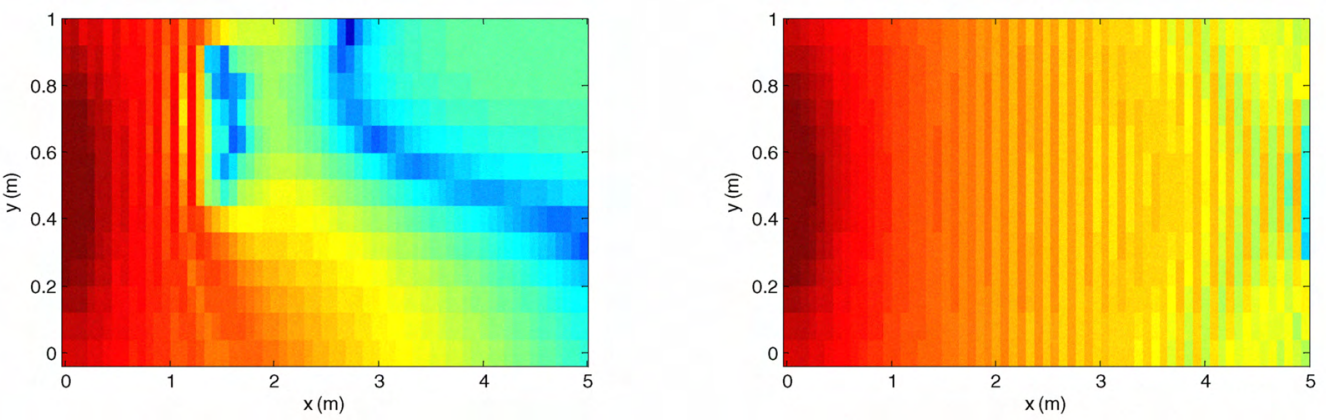

Figure 3 With water bottles (left: at-a-side; right: in front of reader)
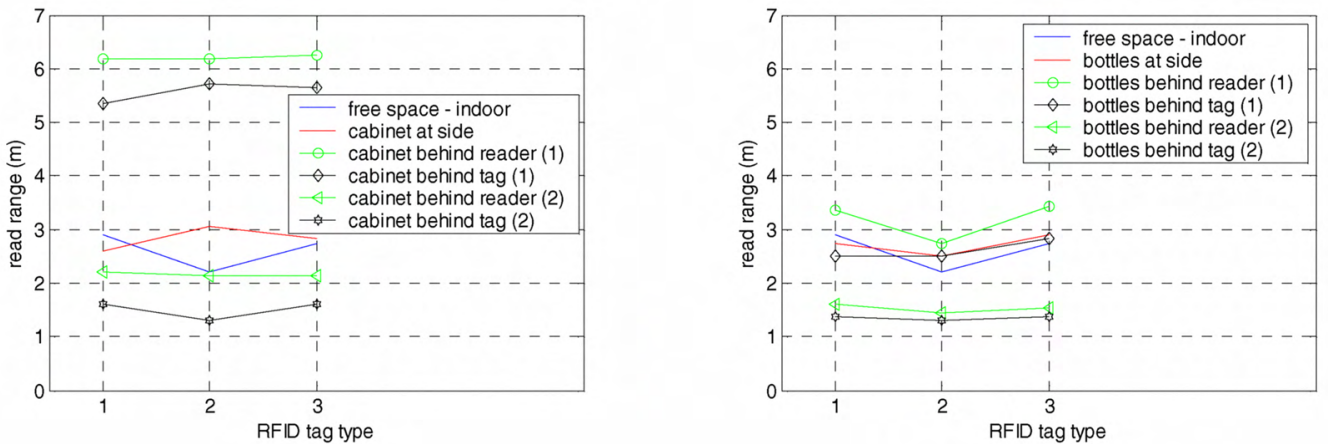

Figure 4 Read range with metallic cabinet (left) and water bottles (right) 